\title{
UN TRAPEZOFORO CON ERACLE E UN'ERMA BIFRONTE CON ERACLE E SILVANO DA VERONA
}

\author{
A TRAPEZOPHOROS WITH HERACLES AND A TWO-FACED HERM WITH HERACLES AND SILVANO \\ IN VERONA
}

\author{
LUIGI QUATTROCCHI \\ Universidad Carlos III de Madrid
}

Lo studio ${ }^{1}$ che si vuol portare all'attenzione si concentra sull'analisi di un gruppo di due sculture romane in marmo, conservate presso il Museo Archeologico del Teatro Romano di Verona.

Questo istituto venne allestito tra il 1919 e il 1923 nel convento dei Gesuiti, collocato sulla riva destra del fiume Adige e adiacente all'antico complesso monumentale. Oltre alle testimonianze e ai reperti ritrovati durante gli scavi dell'edificio antico, il Museo è arricchito da reperti ottenuti con l'acquisizione o la donazione da collezionisti dell'antiquariato locale. Le due sculture che si esporranno in questo lavoro sono comprese in queste due categorie di reperti e si tratta di un trapezoforo acquisito dalla collezione Alessandri di Verona e una doppia erma; le due sculture provengono da vecchie collezioni private, e allo stato attuale è impossibile conoscere il luogo di ritrovamento. Per l'esattezza l'erma bifronte proviene dalla raccolta Giusti, la quale fu smembrata in più parti; il trapezoforo, come accennato, proviene dalla collezione Alessandri; per entrambe le statue, però, non è possibile stabilire con certezza l'arrivo al Museo.

\section{TRAPEZOFORO CON ERMA DI ERACLE}

La prima scultura (Fig. 1), in marmo bianco saccaroide, probabilmente si tratta di marmo lunense, è un trapezoforo (Coarelli, 1966, 968-969) che ha un'altezza massima di $57 \mathrm{~cm}$, una larghezza massima di 34 $\mathrm{cm}$ e uno spessore di $36 \mathrm{~cm}$ (Fig. 2). La scultura si conserva in maniera discreta, sebbene originariamente doveva trattarsi di un'erma bifronte e noi oggi possiamo apprezzarne solo una; nell'unica faccia rimasta sono presenti diverse abrasioni. Il trapezoforo non è inedito, seppur pubblicato in maniera sommaria (Franzoni, 1962, 399; Marchini, 1972, 269); n. d'inventario

1. Si rimanda, per uno studio più generale a C. Rückert (1998) e a H. Wrede (1986).
29531. Presenta un volto realizzato a rilievo, con lo sguardo non frontale ma rivolto a sinistra, per trasmettere una sensazione di stanchezza. Gli occhi risultano incassati con palpebre arcuate, con leggeri fori di trapano per i condotti lacrimali. L'iride è incisa da sottili solchi e le arcate sopraccigliari hanno l'aspetto corrucciato. Si ha l'idea di un volto pensoso, idea che viene aumentata dalla bassa fronte con un'unica ruga centrale. La bocca risulta grande, con il labbro inferiore prominente e quello superiore coperto di baffi, i quali non sono distinti dal resto della barba. I capelli e la barba ricoprono gran parte del viso, unendosi tra di loro ed incorniciando il volto. La capigliatura è resa con ciuffi nodosi, mentre la barba ha piccoli riccioli. Tutto il capo risulta cinto da una corona, probabilmente d'alloro, che ha foglie larghe, resa in maniera appena abbozzata. Tutto il volto risulta comunque eseguito in maniera frettolosa, dando l'idea di un'opera non compiuta, anche se bisogna sempre ricordarsi che si tratta di un supporto, e forse la resa finale di «non concluso» è voluta. L'iconografia è delle più comuni²: il semidio ormai stanco, non più giovane, con l'alloro sul capo a dimostrate l'avvenuta apoteosi, sebbene sia abbastanza raro nella statuaria, e sia invece più utilizzato nei bronzetti, nelle monete e sui camei (Boardmann et alii, 1988, 452, 456, 464, 498, 501, 546; nn. 103, 171, 176, $183,292,779,816,818,822,1527)$ e proprio questa tipologia, dell'Eracle stanco e maturo la troviamo ricorrente nei trapezofori (Marmora Pompeiana, 2008, cat. nn. A 09.).

A livello iconografico e stilistico non si hanno confronti puntuali, anche se questa scultura possiede tratti in comune con ritratti ed erme di un determinato periodo. La tecnica impiegata per rendere l'orbita oculare la ritroviamo alla fine del principato adrianeo, tuttavia, l'incisione dell'iride è più una peculiarità adrianea ed antonina, come sono testimonianza un

2. Per una visione più ampia dell'iconografia erculea si veda: J. Boardmann et alii (1988). 


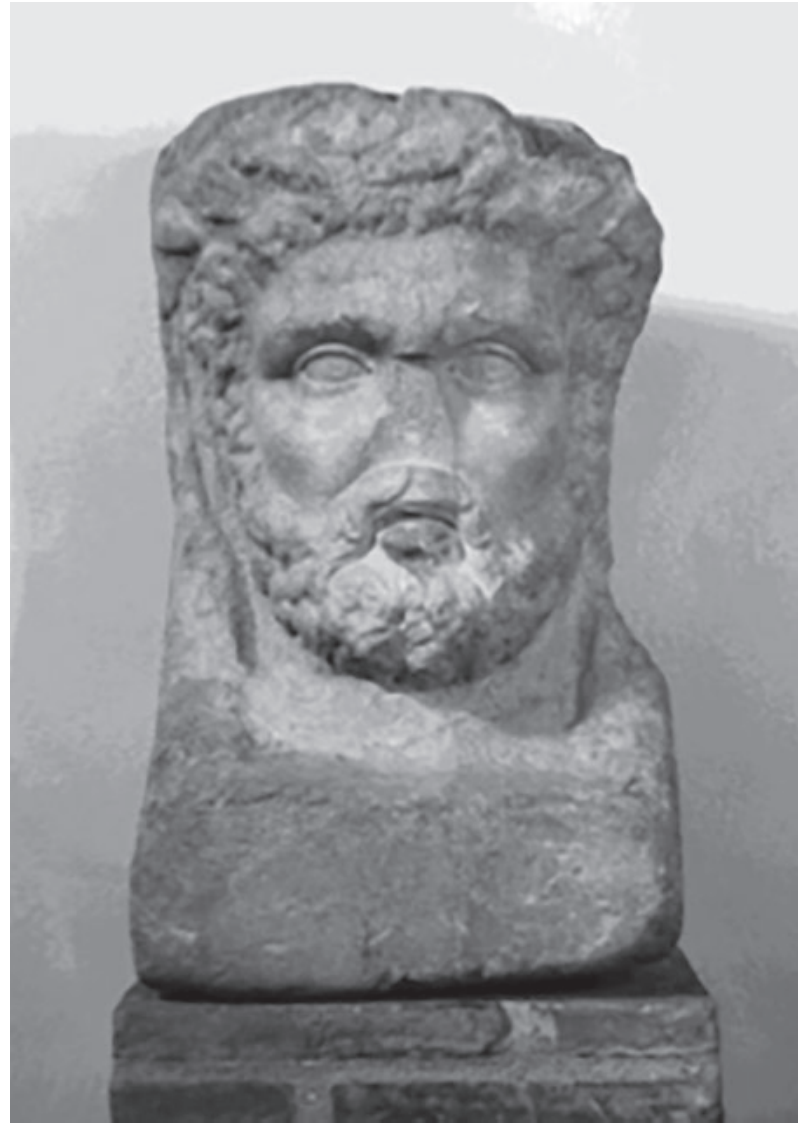

Figura 1: Trapezoforo con erma bifronte di Eracle, Verona $\mathrm{Mu}$ seo del Teatro Romano (Foto per gentile concessione dei Civici Musei di Verona).

ritratto di Antinoo (Fig. 3) (Meyer, 1991, cat. n. 60), tre ritratti di Marco Aurelio che si trovano a Palazzo Colonna, al Museo di Antichità di Bucarest, e al Museo Egizio del Cairo databili tra il 147 e il 160 (Fittschen, 1999, tav. 56, 47, 54), e uno che raffigura Aelio Vero (Fig. 4) (Fittschen, 1999, tav. 119) del 137. La barba ci da un'indicazione temporale importante, perché i riccioli rifiniti con l'uso del trapano sono una prerogativa dell'Eracle Farnese di Napoli, che viene generalmente datato in età severiana (Moreno, 1982, 499). Però il prototipo della nostra scultura sembra rifarsi all'Eracle a riposo (Vermeule, 1975, 323-332), che come le altre statue citate si rifanno a modelli lisippei. Un'ulteriore informazione ce la può fornire la fortuna dei trapezofori a doppia erma, nati presumibilmente nella primissima età imperiale, una grande concentrazione di erme, doppie erme e trapezofori la incontriamo, giusto per fornire un esempio che esuli dal territorio italico, nella Hispania romana, proprio utilizzate all'interno delle villae (Peña Jurado, 2007/2008, 138-141) sebbene qualche studioso affermi che bisognerebbe retrodatare la nascita di questo arredo all'età repubblicana (Wrede, 1985, 5354; Silvestri, 2005, 19-20), periodo nel quale trovano una collocazione nell'arredamento delle ville anche le piccole erme, singole, spesso a tema dionisiaco. Le

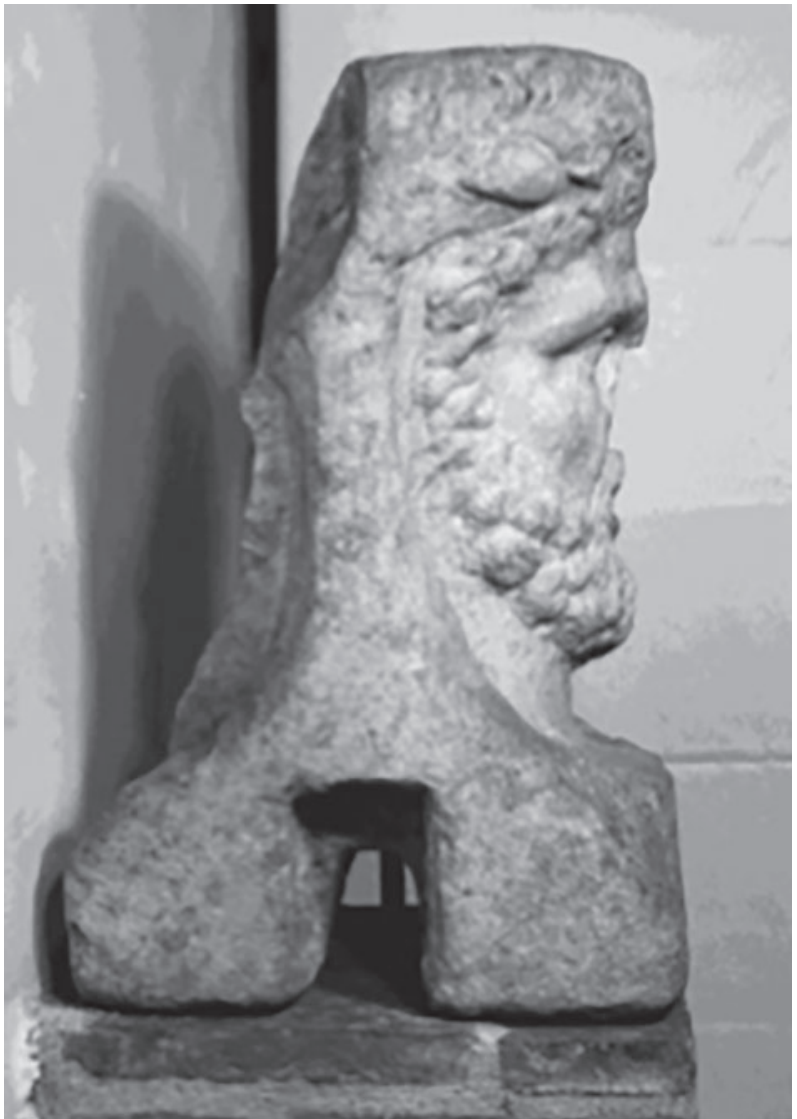

Figura 2: Trapezoforo con erma bifronte di Eracle, Verona museo del Teatro Romano (Foto per gentile concessione dei Civici Musei di Verona).

doppie erme, che raffigurassero filosofi o poeti venivano utilizzate come decoro dei giardini, oltre ovviamente a riflettere l'essenza erudita del proprietario. Ma bisogna annotare che, la nascita di queste doppie erme coincide con l'arrivo a Roma di nuovi culti, spesso di carattere salvifico dell'individuo, e non della comunità come voleva la religione romana. Si potrebbe dunque supporre che le doppie erme assolvessero un doppio utilizzo, sia come ornamento di lusso, sia come oggetto con finalità cultuali, in ambito privato, come per l'appunto l'Eracle domestico. In effetti, le erme doppie o bifronti non sono rare nel mondo artistico sia greco sia romano (Giumlia, 1983, 139), e la doppia rappresentazione trova una certa comodità della natura del semidio. Eracle iniziò, proprio in età adrianea e antoniniana, ad avere una grossa fortuna nell'ambito privato, grazie anche alla sua valenza apotropaica, una tesi molto cara a Moss, che per l'appunto, abbinava il valore apotropaico del nume alla vasta documentazione di trapezofori (Moss, 1988, 271). Si potrebbe dunque avanzare una datazione per questa scultura che non vada oltre i confini della fine del II secolo d.C., infatti l'erma ha un'impostazione stilistica tipica di epoca antonina, con un chiaroscuro molto accentuato e con un uso del trapano per rifinire la capigliatura e la barba. 


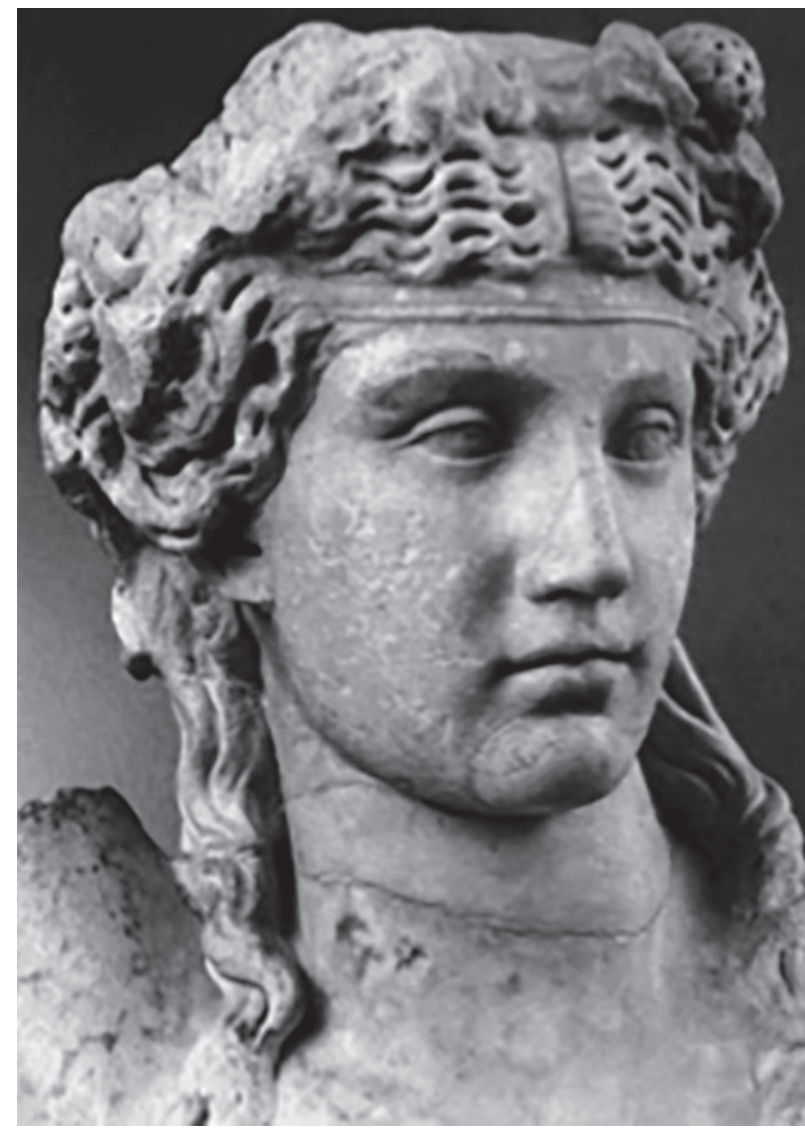

Figura 3: Ritratto di Antinoo, Museo dell'Ontario, Toronto (da Meyer, 1991, tav. 69).

\section{ERMA BIFRONTE CON VOLTO DI ERACLE E SILVANO}

La seconda scultura (Fig. 5), di marmo bianco saccaroide, anche in questo caso è possibile ipotizzare che il marmo provenga dal territorio di Luni, alta $28 \mathrm{~cm}$, è un'erma bifronte con i volti di Silvano ed Eracle. Si presenta in uno stato buono di conservazione, mancante del pilastro su cui si poggiava in origine, piccoli graffi sulla superficie.I volti sono ben preservati sebbene siano evidenti dei guasti nel naso in entrambe le facce. L'erma non è inedita, sebbene mai studiata ampliamente (Riccioni, 1959/1960, 138; Franzoni, 1962, 399), n. d'inventario 29530.

Le erme sono rappresentate sino alla base del collo, uno dei due volti (Fig. 6) sembrerebbe avere delle inflessioni arcaistiche, vista la resa della capigliatura e della barba: la capigliatura è resa da ciocche di forma uncinata, chiara ispirazione severa, eseguita in maniera sommaria e frettolosa, ricade sulla fronte liscia, che a sua volta è tondeggiante e ampia; un profondo foro di trapano ricrea il ricciolo della parte terminare, mentre il resto della capigliatura è appiattito e semplicemente distinta da piccoli e leggeri solchi ondulati. La barba ripropone la trattazione sintetica e superficiale riscontrata nella frangia, dalla quale riprende il

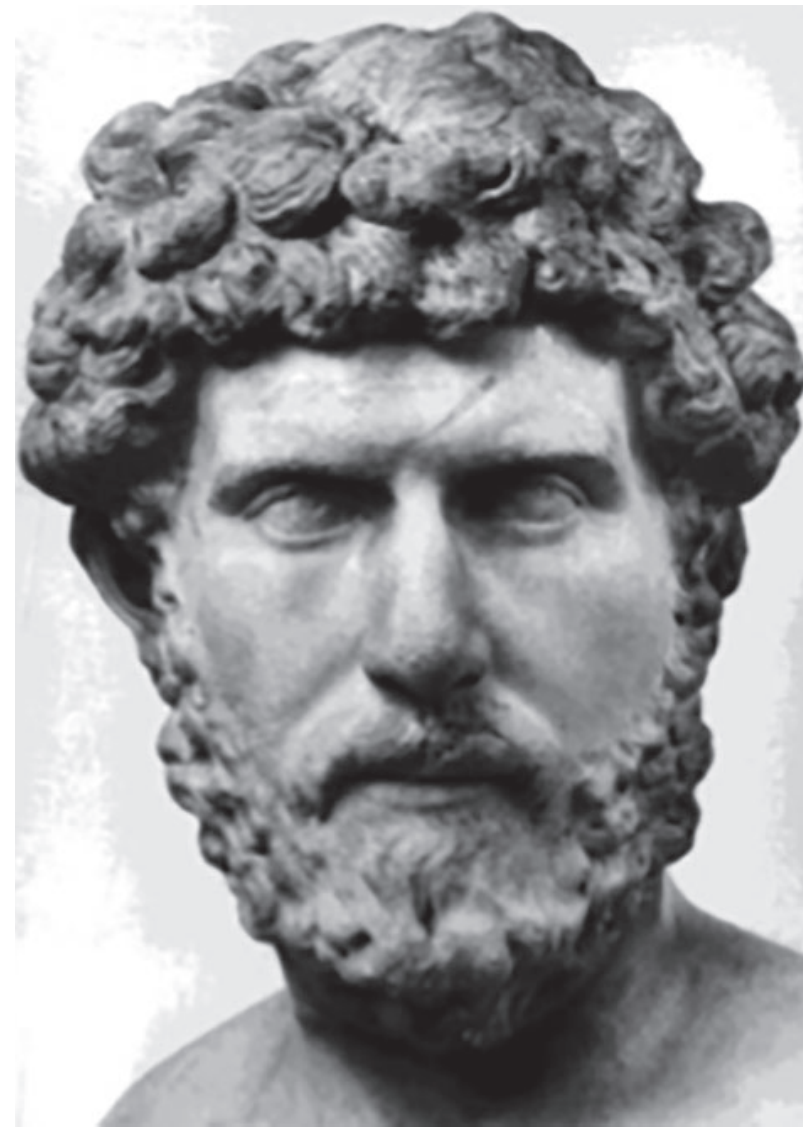

Figura 4: Ritratto di Aelio Vero, Petworth, slg. Leconfield (da Fittschen, 1999, tav. 119).

medesimo accorgimento nella definizione dei riccioli terminali, così che il risultato finale si traduce in una massa di ciocche simmetriche, non differenziate e alquanto abbozzate. Due piccoli baffi incorniciano il labbro superiore; la bocca è piccola e socchiusa, con labbra sporgenti e affusolate, divise da un ondulato solco. Il volto è ovale e neutro, privo di qualsiasi tratto fisiognomico con superfici levigate. Le orecchie sono piccole con padiglioni stretti, con un piccolo foro nella parte interna. Gli occhi sono a mandorla, dal bulbo sporgente, resi da uno stampo di tipo classico, con palpebre lamellari. Le caruncole lacrimali sono realizzate da un unico foro di trapano. Il capo risulta cinto da una corona di pino, molto schematizzata, in cui i ciuffi di aghi sono riconoscibili a stento, tripartiti in punte all'estremità, probabilmente ad essi si alternano delle pigne stilizzate, di forma cilindrica, che tengono uniti i gruppi di foglie. Le due tenie che costituiscono le estremità della fascia avvolgente il capo sono annodate ai lati.

Le due erme sarebbero identiche se non per delle piccole differenze: la seconda erma (Fig. 7) ha la barba corta, composta da boccoli approssimativi; la capigliatura è costituita da ciocche corte ad archetto; il labbro superiore è coperto da baffi spioventi, abbozzati, mentre a differenza dell'erma «arcaistica» la fronte 


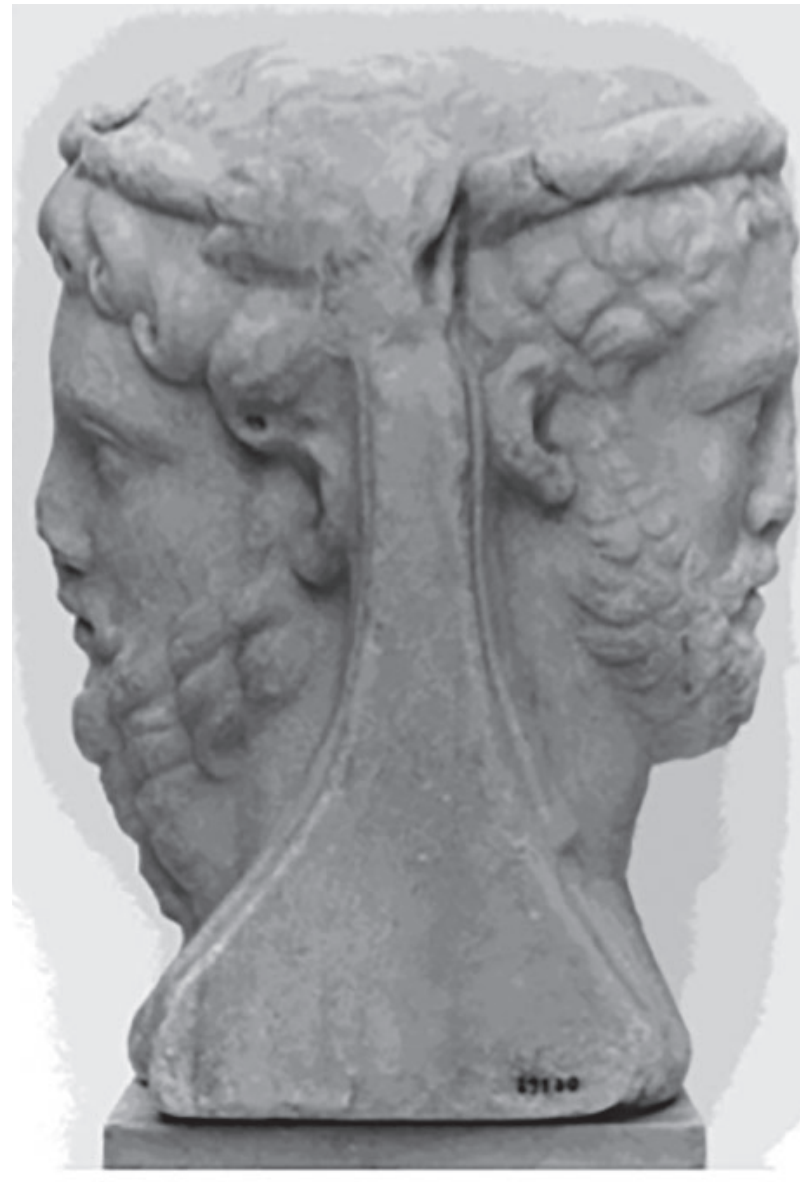

Figura 5: Doppia erma di Silvano ed Eracle, Museo del Teatro Romano di Verona (Foto per gentile concessione dei Civici Musei di Verona).

è solcata in maniera orizzontale da un'unica ruga. Il capo è cinto da uno strophium, un simbolo di apoteosi, e si risolve per ciascun lato del volto con una doppia tenia (Aurigemma, 1930).

La tipologia monumentale del pezzo veronese è dunque riconoscibile come un'erma bifronte neo-attica (Silvestri, 2005). Si tratta di un'invenzione romana, con origini che affondano al I secolo d.C., anche se si ipotizza un inizio della produzione in tarda età repubblicana, e che viene a mancare durante il IV secolo d.C. (Silvestri, 2005, 19-20; Giumlia, 1983, 20-24). Queste sculture a duplice soggetto sembrano restare confinate solo nelle abitazioni private come domus o villae. Le ritroviamo sempre in contrapposizione mai affiancate e non necessariamente i personaggi sono differenti, anche se spesso si ritrovano in chiasmo, ovvero un imberbe su una faccia e un barbato sull'opposta (Wrede, 1986, 53-54); ulteriore contrapposizione è quella data dal sesso: una figura femminile da un lato e una maschile dall'altro, come nell'erma di Eracle e Onfale di Treviso (Galiazzo, 1982, 104-107).

Il tipo della nostra scultura è di tipo «paterno» (Silvestri, 2005, 28-29), con la contrapposizione di due volti barbati pertinenti a due personaggi differenti. Il linguaggio arcaistico è decisamente presente

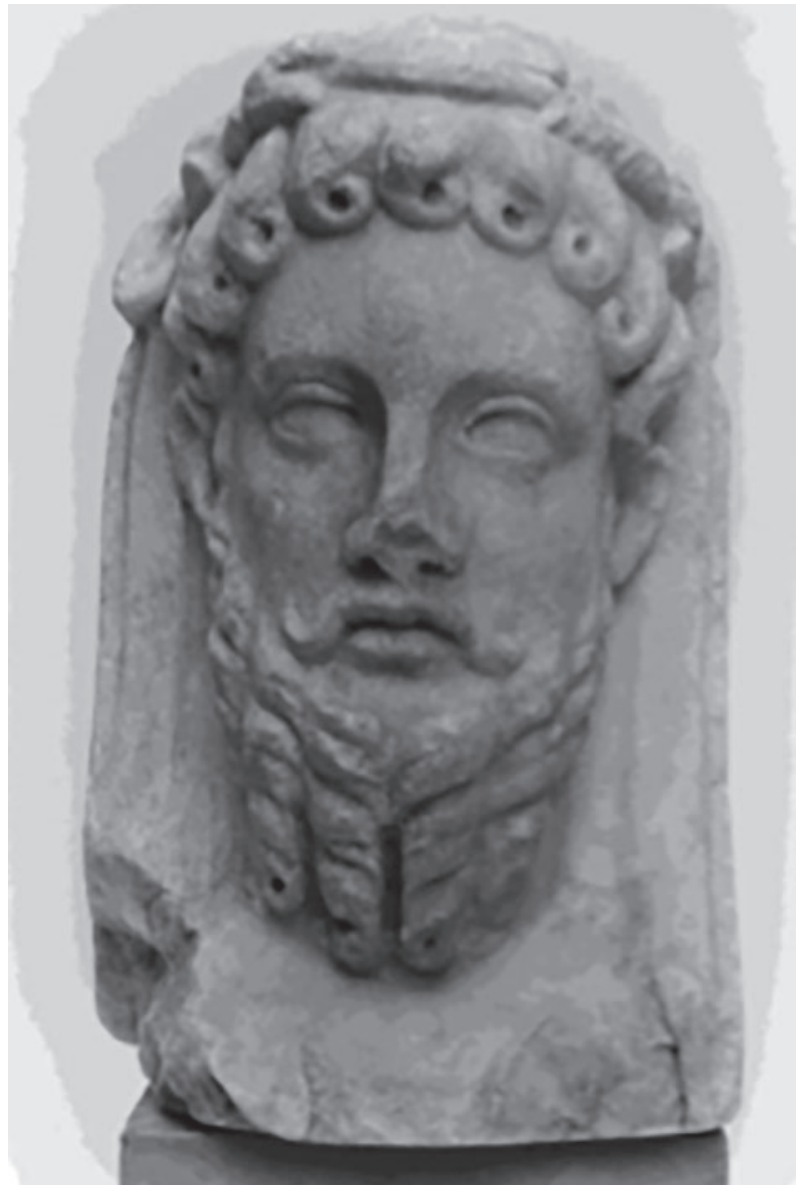

Figura 6: Doppia erma di Silvano ed Eracle, Museo del Teatro Romano di Verona (Foto per gentile concessione dei Civici Musei di Verona).

nell'elaborazione di capelli e barba, nella prima erma, neutra e ovale in entrambi i volti. Ma non mancano i tratti classici: occhi con palpebre lamellari, volti luminosi e levigati. Non deve stupiste questa commistione di iconografie e stili, perché non è affatto un fenomeno raro (Fullerton, 1990, 8). Questa scultura «gianiforme» si presenta come un lavoro curato, con stile ricercato, sebbene sia reso in maniera semplicistica e schematizzata, probabilmente per le ridotte dimensioni della scultura $(28 \mathrm{~cm})$. Iconograficamente si avvicina ai modelli del V secolo a.C., ma nella scelta dei soggetti si distacca dai tipi canonici, rivelando probabilmente una connotazione molto più provinciale, $\mathrm{o}$ meglio ancora, locale.

\section{IDENTIFICAZIONE DEI PERSONAGGI}

Per l'identificazione dei soggetti rappresentati ci viene in aiuto la comparazione iconografica, con esemplari simili e non sempre stilisticamente congruenti.

L'erma con lo strophion è riconducibile a Eracle (Boardmann, Palagia y Woodford, 1988, 728-838), e l'iconografia riprende tipiche rappresentazioni del semidio, nella sua versione barbata (Galliazzo, 1982, 


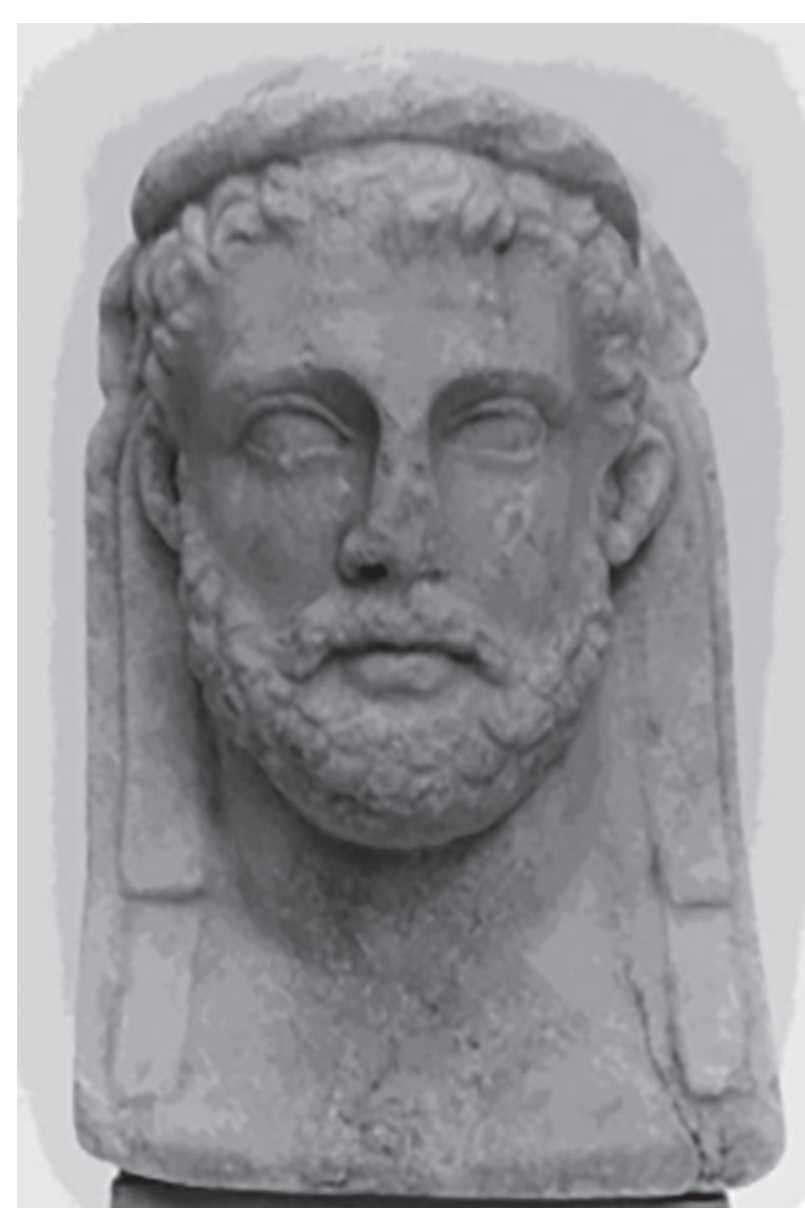

Figura 7: Doppia erma di Silvano ed Eracle, Museo del Teatro Romano di Verona (Foto per gentile concessione dei Civici Musei di Verona).

104-107), ormai adulto. Con lo strofio lo ritroviamo in diverse produzioni statuarie (Boardmann, Palagia y Woodford, 1988, figs. 186, 189, 209, 625, 638, 653, $721,785,809,824,970,973,1143$ ). Per un confronto stilistico ed iconografico invece possiamo addurre tre esempi: una testa a Palazzo Medici Riccardi (Fig. 8) che viene generalmente datata agli inizi dell'epoca imperiale (Saladino, 2000, 357-358), una testa dalla gliptoteca di Copenaghen (Fig. 9) del III secolo a.C. (Poulsen, 1951, 189-190), e infine un trapezoforo situato al museo di Coo (Laurenzi, 1957, 136). A mio avviso il confronto più stringente si ha con la testa di Copenaghen, per lo meno dal punto di vista stilistico ed iconografico: gli occhi dal taglio classico, la stilizzazione della barba, e l'uso del trapano (sebbene nel pezzo veronese il trapano sia usato in maniera molto più pesante ed evidente), mentre gli altri pezzi si accostano per analogie soprattutto sui capelli e sulla barba.

La fisionomia dell'erma arcaica ci rimanda a confronti con l'iconografia dionisiaca, in particolare un esemplare (Fig. 10) conservato al Museo Archeologico Nazionale di Napoli (Moss, 1988, 559), insieme ad un altro esemplare situato presso i Musei Vaticani, datato al I secolo d.C. (Lippold, 1956, 107). In termini di qualità, i pezzi appena citati, trovano una notevole

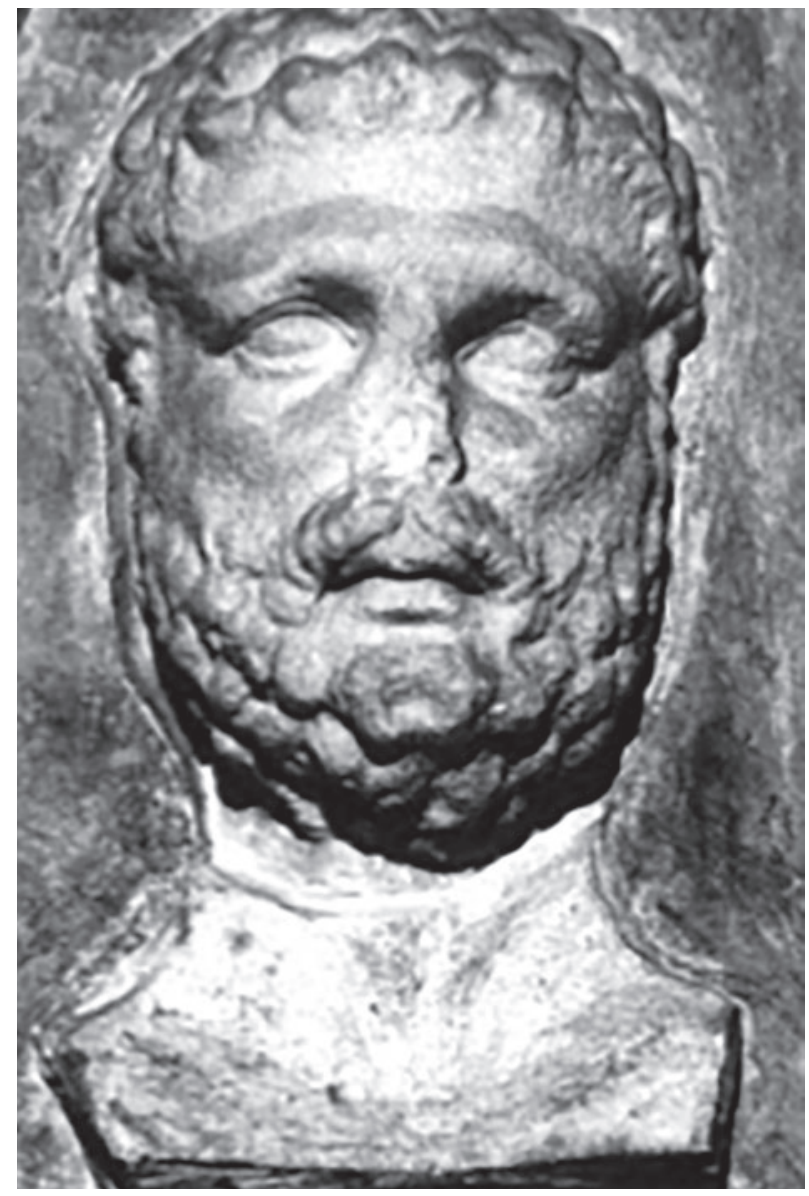

Figura 8: Testa di Eracle, Palazzo Medici, Firenze (da Saladino, 2000, tav. 146, VI.2).

difficoltà ad accostarsi al frammento veronese, ma il taglio arcaistico della capigliatura e della barba richiamano formalmente l'erma coronata di pino. Per una stessa fattura stilistica della barba, confronti più convincenti si ritrovano in due doppie erme, rappresentanti Dioniso, la prima (Fig. 11) si ritrova anch'essa presso il Museo Archeologico Nazionale di Napoli (Marmora Pompeiana, 2005, 119-120), mentre la seconda, del I secolo d.C., al Museo di Nyon (Bossert, 2002, tav.1). In questi due esempi l'uso del trapano è quasi inesistente, iniziando a suggerirci un'anticipazione rispetto alla cronologia della nostra erma.

Dai confronti sembrerebbe opportuno presupporre che l'erma arcaistica riproduca le fattezze di Dioniso $^{3}$ (Veneri y Gasparri, 1986, 432): l'iconografia parrebbe comprovata anche dalla sua combinazione con Eracle, frequentemente associata al fratellastro. Nelle province iberiche ${ }^{4}$ troviamo un'erma arcaistica, che rappresenta Dioniso, ora conservata nel Museo

3. Per l'iconografia dionisiaca si veda: C. Gasparri y A. Veneri (1986).

4. Per uno studio più approfondito si guardi: A. Peña Jurado (2002). 


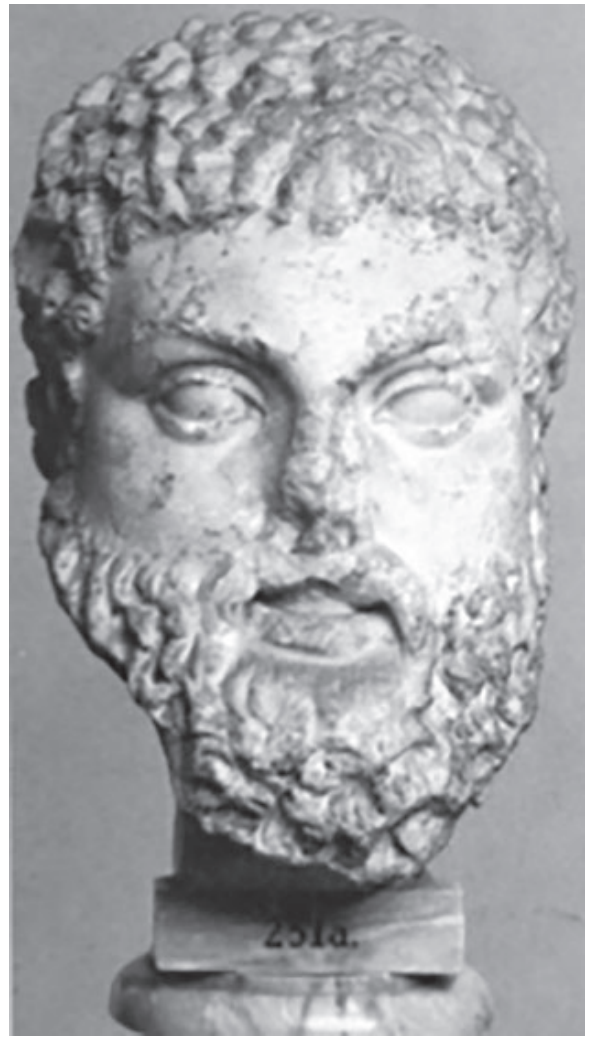

Figura 9: Testa di Heracles, Ny Carlsberg Glyptoteche, Copenaghen (da Boardmann, Palagia y Woodford, 1988, n. 442).

Histórico Municipal de Écija, datata all'età neroniana-flavia, che sebbene abbia tratti arcaistici (Peña Jurado, 2004, 279-281, lamina VII) si discosta, almeno per quanto riguarda la trattazione del marmo, dalla nostra scultura.

Però l'opera analizzata ha un attributo non diffuso nel mondo scultoreo, e riservato ai soli personaggi della natura selvatica: la corona di pino. La particolarità di quest'ornamento risiede principalmente nella sua realizzazione, che non è confrontabile con la sua iconografia più tipica. Infatti, quest'ultima prevede ciuffi di aghi disposti e collegati uno di seguito all'altro, mentre nel nostro esemplare, oltre alla notevole semplificazione, gli aghi risultano compattati in un unico mazzo a tre punte, sono praticamente indistinguibili se non attraverso leggeri solchi. Confronti stringenti li troviamo in una collezione privata (Fig. 12) di Franz Graf a Erbach (Wrede, 1972, 82-83) e uno alla Ny Carlsberg Glyptotec di Copenaghen (Poulsen, 1951, 104-105) il quale rappresenta un atleta. Se quindi il riconoscimento della corona di pino fosse corretta, apparirebbe insolito che la ritrovassimo nel capo di un Dioniso barbato.

Il pino è un attributo che in epoca arcaica veniva associato al dio del simposio, ma per adesso le uniche corone che ritroviamo sopra il capo di Dioniso solo solo di vite o edera. Ma nell'erma veronese l'intento era di connotare una differente divinità, ovvero Silvano (Paci, 2000, 163).

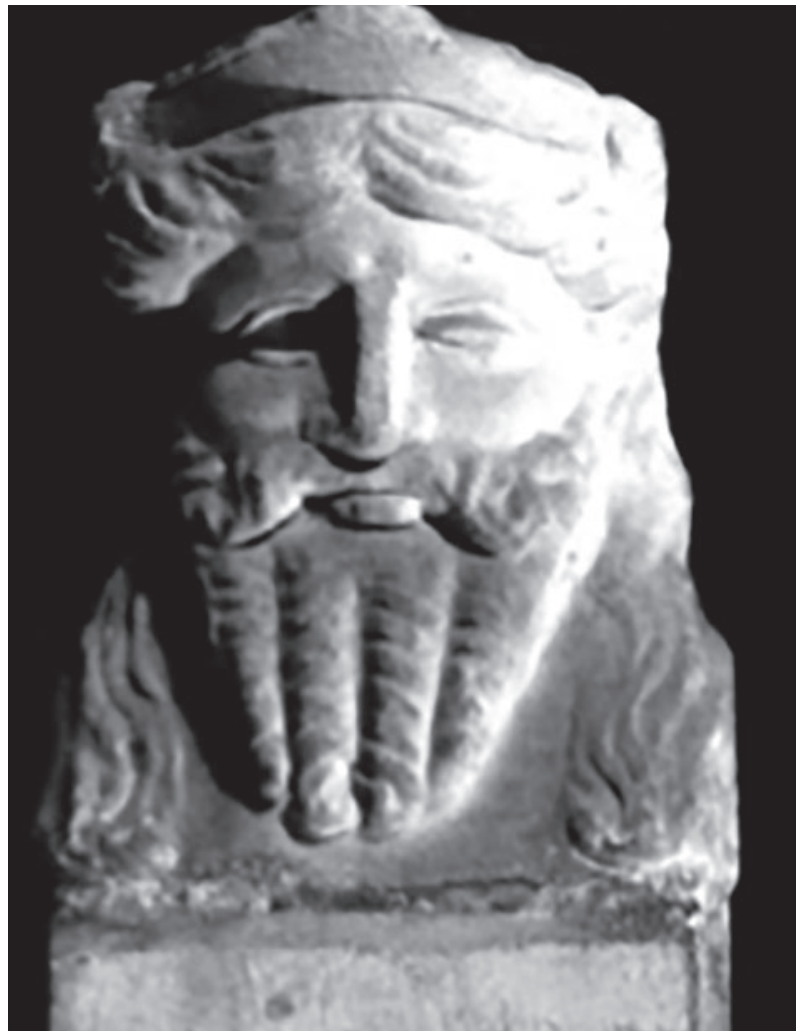

Figura 10: Erma di Dioniso, Museo Archeologico Nazionale di Napoli (da Marmora pompeiana, 2005, cat. n. C08, p. 120).

Silvanus è una divinità di origine italica, di cui si conserva la memoria anche in epoca medievale (Emacora, 2013, 665-676), legato soprattutto all'ambiente selvaggio delle foreste, adorato dagli strati sociale più umili come liberti, schiavi, indigeni e soldati. Questa sua caratteristica, abbinata a alcune somiglianze iconografiche, come la nudità e la corona di pino, fanno in modo che spesso venga confuso con Faunus (Pouther y Rouillard, 1997, 582-583) o addirittura assimilato al dio Pan (Marquardt, 1995, 923-941). Le tre divinità però fanno parte di realtà culturali differenti, sebbene in alcune zone si possa notare un certo sincretismo (Dorcey, 1992, 42). La maggiore tendenza a ritrarre Fauno e il dio greco Pan con tratti caprini (Dzino, 2012, 265) è una delle differenze principali, constatando che invece Silvano ${ }^{5}$ mantiene un aspetto umano e benevolo.

La somiglianza tra questa rappresentazione di Silvano e le effigi di Dioniso non è però un fenomeno strano; lo stile arcaistico impiegato in raffigurazioni di Bacco venne, in epoca romana, ampiamente esteso a creature e divinità della sua cerchia, o comunque a culti essenzialmente rustici (Fullerton, 1990, 560570). La scelta di rappresentare Silvano con un'iconografia dionisiaca può quindi ricondursi alla sua

5. Per quanto concerne l'iconografia di Silvano si guardi: A. M. Nagy (1994). 


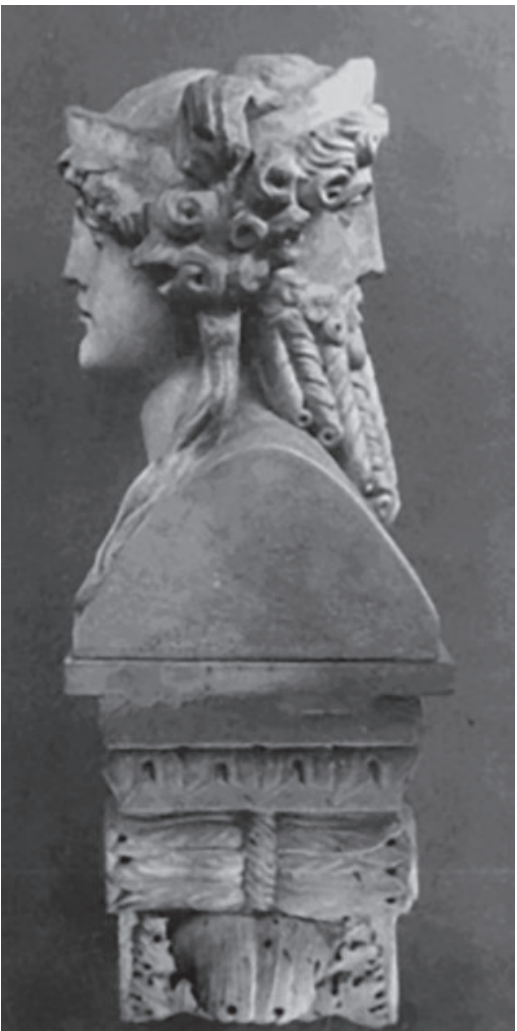

Figura 11: Erma di Dioniso, Museo Archeologico Nazionale di Napoli (da Marmora pompeiana, 2005, cat. n. C08, p. 120).

appartenenza al thiasos dionisiaco, ma senza escludere l'associazione Liber-Silvano (Matijević y Kurilić, 2011, 152-155).

Liber (Matijecić y Tassaux, 2000, 67-72) è una divinità italica presente nel Lazio già nel V secolo a.C., estranea però a quei territori permeati di cultura greco/ ellenistica, una divinità collegata all'ambiente rustico, protettore dei pastori e delle greggi, e in età più tarda degli schiavi (Poulsen, 1951, 351-352). Il suo culto non si distribuì omogeneamente in tutto l'Impero, trovò consenso in Pannonia, Dacia e in Africa, molto più limitato è in Gallia e Hispania.

Nonostante Liber si configurasse come il corrispettivo italico di Dioniso (Homann Wedeking, 1958, 114), il suo culto rimaneva privo di quell'aspetto misterico e orgiastico del rituale dionisiaco.

Il culto Liber-Bacco ottenne notevole considerazione dalla fine del I secolo d.C., propagandato soprattutto da imperatori antonini e severi. In questa fase l'assimilazione delle divinità era ormai completa, tanto da rendere indistinguibili iconograficamente i due dei (Matijecić y Tassaux, 2000, 76).

L'unione di Silvano con Liber si affermò durante l'età antoniniana e severa, in zone come l'Italia e la Dalmazia, ma un primo vincolo religioso lo si trova anche nella prima età imperiale (Matijević y Kurilić, 2011, 156-157). Principalmente l'unione avvenne sulla ritualità della fertilità e delle varie attività agro-pastorali, anche se ciascuno dei due mantenne la propria

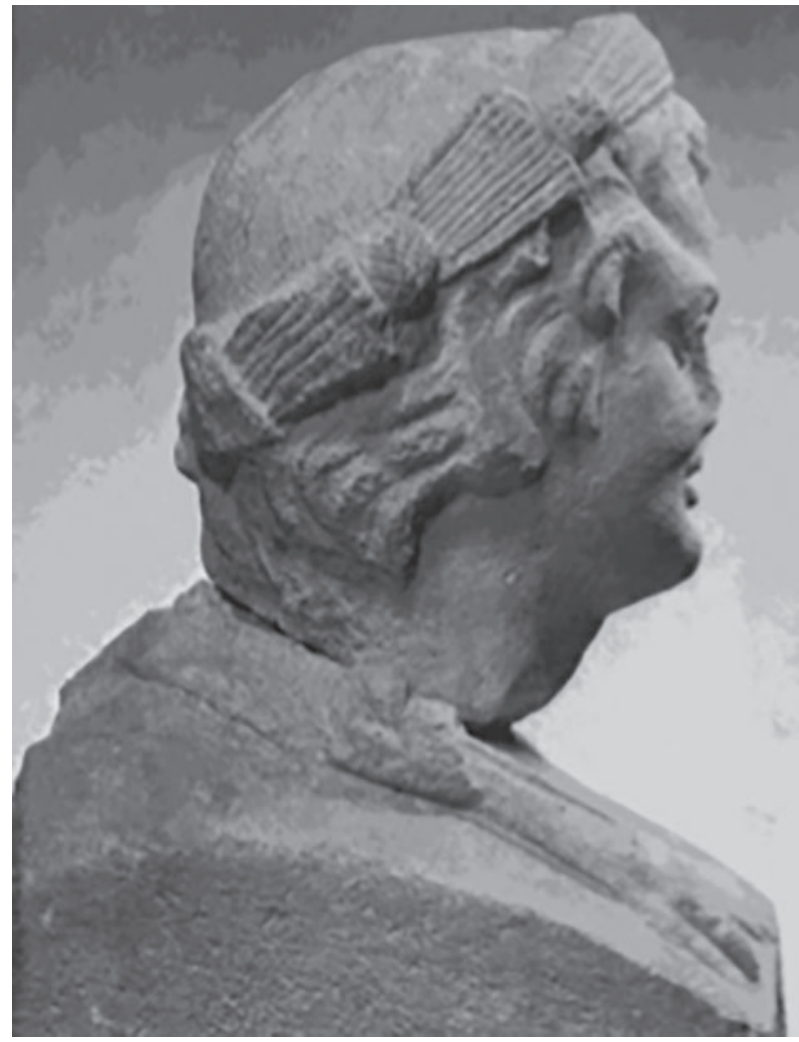

Figura 12: Erma di Satiro con corona di pino, Hermengalerie, Welschbillig (da Wrede, 1972, tav. 48-49).

connotazione originaria (Rendić Mioćević, 2007, 35-39).

Nel volto arcaicizzante della doppia erma barbata è quindi riconoscibile il dio Silvano e la sua iconografia, così simile alle raffigurazioni arcaiche di Dioniso, è probabilmente riconducibile alla sua pertinenza al thiasos bacchico, oltre che alla sua identificazione, in alcune regioni, alla controparte italica del dio del vino, Liber Pater. La sua associazione con il semidio Eracle, pertanto, non ci sorprende, in quanto egli stesso godette di ampia fortuna in tutta la Cisalpina. La sua presenza insolita, ma non di difficile comprensione o priva di un significato ideologico, quale alter ego di una divinità della foresta come Silvano, è verosimilmente imputabile alla caratteristica del nume greco di protettore della pastorizia o della transumanza (Giumlia, 1983, 145).

Le due divinità trovano tra loro una connessione anche su altri piani di comprensione; si tratta di una combinazione che per gli antichi dovette essere comunque immediata, soprattutto in quelle regioni che basavano il proprio sostentamento e la propria fortuna su attività di questo tipo. L'associazione tra Eracle e Silvano trova nessi anche a livello mitologico, infatti, le due divinità sono effettivamente correlate da un legame di parentela, esiste un'iscrizione in cui il nume greco è definito nipote di Silvanus:

Hercules Inuicte sancte Siluani nepos/ hic (sic) adunisti ne quid fiat mali (CIL VI, 30738; Emacora, 2013, 668). 
Inoltre entrambi sono divinità legate alla fertilità maschile, con rituali escludi alle donne (Bassignano, 1987, 343), sono garanti dei confini (Panciera, 2005, 433 ) ed entrambi molto presenti nel culto domestico, con un valore apotropaico.

\section{DATAZIONE}

La datazione in età adrianea è formulata in base ai confronti sopra esposti e su caratterizzazioni stilistiche generiche osservate in esemplari di questo periodo. In base al trattamento di capigliatura e barba e all'uso ricorrente, ma non eccessivo, del trapano corrente, il pezzo sembra ben inserirsi nel clima artistico circoscritto al principato adrianeo o su sulla sua scia in epoca antoniniana. Si è osservato come in tale periodo si registra un notevole interesse verso quelle opere scultoree ispirate ai temi e alle forme dello stile arcaico, parallelamente al ricorso ai tipi dei modelli neoattici (Fullerton, 1990, 194-195), inoltre è anche in questo clima culturale che il culto di Silvano ebbe una certa fortuna. Trattandosi di una datazione su base prettamente stilistica è possibile considerare un margine di errore, tuttavia la doppia erma veronese è ascrivibile non oltre il II secolo d.C.

\section{CONCLUSIONI}

A livello di documentazione, la zona veronese conta alcune testimonianze circa il culto di Eracle, sia su base epigrafica sia scultoria. La scultura non permette neppure di trarre nuove considerazioni in merito a un'eventuale derivazione autoctona del culto di Eracle in Cisalpina. La teoria finora più comprovata suggerirebbe un'introduzione del culto tramite le popolazioni centro-italiche trasferitesi a nord nell'instaurazione o nel ripopolamento delle nuove colonie romane; di minore portata sarebbe, invece, il contributo dei culti locali di substrato celtico o etrusco (Pascal, 1964, 164$165)$. In contrapposizione abbiamo notevoli testimonianze sul culto di Silvano: un rilievo (Franzoni, 1962, 399-400), due teste marmoree raffiguranti il dio (Roscher, 1909, 833) e otto epigrafi (CIL V, 3295; 3296; $3297 ; 3298 ; 3299 ; 3300 ; 3301 ; 3302)$, distribuite in area urbana e extra-urbana. I dedicanti sono tutti di livello sociale medio-basso, liberti o schiavi, e tra di essi si nota un cacciatore a cui vennero conferiti gli ornamenta decurionali (CIL V, 3302).

Proprio per la sua natura silvicola il culto di Silvanus veniva celebrato in aree boschive, a confine tra il territorio urbano ed extraurbano, con zone devozionali, idoletti in legno e piante a lui dedicate (Zaccaria, 2000, 1109-1115). La dislocazione nell'agro extramurario e l'assenza dell'epiteto domestico suggerirebbero, pertanto, l'esistenza a Verona di una ritualità silvana ancora legata all' ambito «selvaggio» delle zone boschive e inerente alla protezione della pastorizia. Tuttavia la tipologia della nostra erma, contraddittoriamente alle documentazioni finora esaminate, sembra porre il culto del dio proprio in una situazione domestica/privata. Potrebbe quindi, in linea generale, sancire una fase in cui a Verona il culto si spostò nell' area intra-urbana.

Nonostante la destinazione solitamente domestica del supporto, conservante le effigi di Eracle e Silvano, e considerando che spesso queste sculture erano destinate a decorare aree aperte proprio per una funzione tutelare e apotropaica, non è sconcertante l'idea che la nostra erma gianiforme potesse essere destinata a ufficializzare un boschetto sacro destinato alla venerazione di Silvanus e Hercules. La diffusione del culto di Eracle e Silvano e le conseguenti implicazioni nel campo delle attività di allevamento/commercializzazione della lana non smentiscono una possibile appartenenza dell'erma al territorio di Verona, ma, malgrado tutte queste premesse, non possiamo con tale sicurezza attribuire la nostra erma a un ipotetico luogo di culto sul locale. Importante è la sua testimonianza quale prova di una devozione singolare, che congiunge due divinità certamente connesse a una società improntata su attività di tipo agro-pastorali. Sulla reale identità di questa cultura locale possiamo solo limitarci a mere speculazioni, in attesa di dati più comprovanti. Di sicuro la nostra erma sembra inserirsi bene nel quadro cultuale e culturale della Verona romana, ma, anche senza allontanarci troppo, non sono pochi gli altri insediamenti sul territorio della Venetia et Histria, che si conformerebbero altrettanto bene come possibili candidate dell'origine del reperto, fra tutte la stessa Aquileia (Murgia, 2013, 275-280).

\author{
Luigi Quattrocchi \\ Via De Amicis 1 \\ 09013 Carbonia (Italia) \\ 1.quattrocchi@hotmail.it
}

\section{BIBLIOGRAFÍA}

AURIGEMMA, S., 1930: s. v. «Benda», in Enciclopedia Italiana di Scienze, lettere ed Arti, VI, 597-598, Roma.

BASSIGNANO, M.S., 1987: «La religione: divinità, culti, sacerdozi», in E. Buchi (a cura di), Il Veneto nell'età romana, I, Storiografia, organizzazione del territorio, 343, Verona.

BOARDMANN, J., PALAGIA, O. y WOODFORD, S., 1988: V «Herakles», LIMC, IV, 728-838, Zürich-München.

BOSSERT, G., 2002: Die figürlichen Skulpturen von Colonia Julia Equestris, Lausanna.

COARELLI, F., 1966: s.v «Trapezoforo», EAA, VII, 968969, Roma.

DORCEY, P.F., 1992: The cult of Silvanus, A study in Roman Folk Religion, Leiden-New York-Köln.

DZINO, D., 2012: «The cult of Silvanus: rethinking provincial identities in Roman Dalmatia», VjesAMuzZagreb, XLV, 261-279.

EMACORA, D., 2013: «Sanctus Silvanus, Homo Selvaticus; la ripresa del sacro selvaggio?», in S. Magnani (a 
cura di), Studi di frontiera, I: Le aree montane come frontiere. Spazi d'interazione e connettività, Atti del Convegno Internazionale (Udine, 10-12 dicembre 2009), 665-676, Roma.

FRANZONI, L., 1962: «Il culto di Silvano a Verona», Vita Veronese, XV, 399-402.

FITTSCHEN, K., 1999: Fittschen, Prinzenbildnisse antoninischer Zeit, Mainz am Rhein.

FULLERTON, M.D., 1990: The Archaistic Style in Roman Statuary, Leiden-New York-København-Köln.

GALLIAZZO, V., 1982: Sculture greche e romane del Museo Civico di Treviso, Roma.

GASPARRI, C. y VENERI, A., 1986, «Dionysos», Limc, vol.III, 1, 414-514, Zürich-München.

GIUMLIA, A., 1983: Die neuattischen Doppelhermen, Wein.

HOMANN WEDEKING, E., 1958: v. «Dioniso», EAA, III, 112-115, Roma.

LAURENZI, L., 1957: «Sculture inedite del Museo di Coo», AnnScAt, XXXIII-XXXIV, n. s. XVII-XVIII, p. 106 ss.

LIPPOLD, F., 1956: Die Skulpturen des vaticanischen $\mathrm{Mu}$ seums, Berlin.

MARQUARDT, N., 1995: Pan in der hellenistischen und kaiserzeitlichen Plastik, Bonn.

MATIJAŠIĆ, R. y TASSAUX, F., 2000: «Liber et Silvanus», in Ch. Delplace y F. Tassaux (a cura di), Les cultes polythéistes dans l'Adriatique, Ausonius Études IV, 65118, Bordeaux.

MATIJEVIĆ, I. y KURILIĆ, A., 2011: «Two unpublished altars in Salona», OpArch, XXXV, 133-167.

MEYER, H., 1991: Antinoos; die achäologischen Denkmäler unter Einbeziehung des numismatischen und epigraphischen Materials sowie der literarischen $\mathrm{Na}$ chrichten, München.

MOSS, C.F., 1988: The roman marble tables, Princeton.

MURGIA, E., 2013: Culti e romanizzazione: resistenze, continuità, trasformazioni, Trieste.

NAGY, A. M., 1994: «Silvanus», LIMC, VII, 763-773, Zürich-München

PACI, G., 2000: «I culti pagani sulle due sponde dell'Adriatico centrale», in Ch. Delplace y F. Tassaux (a cura di), Les cultes polythéistes dans l'Adriatique, Ausonius Études IV, 155-165, Bordeaux.

PANCIERA, S., 2005: «Silvano a Roma», in S. Panciera (a cura di), Epigrafi, Epigrafia, Epigrafisti. Scritti editi e inediti (1956-2005) con note complementari e indici, I, 421-437, Roma.
PASCAL, C.B., 1964: «The Cults of Cisalpine Gaul», Latomus, LXXV, 170-178.

PEÑA JURADO, A., 2002, Hermas de pequeño formato del Museo Arqueológico de Córdoba, Córdoba.

PEÑA JURADO, A., 2004: «Nuevos hermas de pequeño formato de la Bética», Anales de Arqueologia Cordobesa, 15, 271-289.

PEÑA JURADO, A., 2007/2008: «La escultura de la domus en Hispania», AnMurcia, 23-24, 119-144.

POULSEN, F., 1951: Catalogue of Ancient Sculpture in the Ny Carlsberg Glyptotek, Copenaghen.

POUTHIER, P. y ROUILLARD, P., 1997: v. «Faunus», LIMC, VIII, 582-583, Zürich-Düsseldorf.

RENDIĆ MIOČEVIĆ, A., 2007: «Sylvanus of the Delmatae and traces of cult syncretism with Liber», HistAnt, XV, 33-43.

ROSCHER, W.H., 1909: Ausfuhrliches Lexikon der griechischen und romischen Mythologie, vol. IV, Leipzig.

RÜCKERT, C., 1998, «Miniaturhermen aus Stein. Eine Vernachlässigte Gattung kleinformatiger Skulptur der römischen Villeggiatur», $M M, 39,176-237$.

SALADINO, V., 2000: Le antichità di Palazzo Medici Riccardi II: Le sculture, Firenze.

SILVESTRI, A., 2005: Le erme bifronti di Aricia, Ippolito-Virbio e $i$ riti arcaici di iniziazione, Roma.VENERI, B.A. y GASPARRI, C., 1986: v. «Dionysos», LIMC, III, 413-566, Zürich-München.

VERMEULE, C., 1975: «The Weary Herakles of Lysippos», AJA, CXXIX, 323-332.

WREDE, H., 1972: Die Spätantike Hermengalerie von Welschbillig: Untersuchung zur Kunsttradition im 4. Jahrhundert $n$. Chr. und zur allgemeinen Bedeutung des antiken Hermenmals, Berlin.

WREDE, H., 1986: Die antiken Herme, Mainz am Rhein..

ZACCARIA, C., 2000: «Una familia Silvani sul colle di S. Giusto a Trieste», in G. Paci y L. Gasperini, (a cura di), Epigraphai. Miscellanea epigrafica in onore di Lidio Gasperini, 1099-1117, Tivoli.

\section{ABBREVIAZIONI}

$\mathrm{CIL}=$ Corpus Inscriptionum Latinarum, Berlin 1863-

MARMORA POMPEIANA 2008 = CARELLA, A., D'ACUNTO, L.A., INSERRA, N. y SERPE C. (a cura di), Marmora pompeiana nel Museo Archeologico Nazionale di Napoli. Gli arredi scultorei delle case pompeiane, Roma. 